\title{
Bocavirus humano en Chile: características clínicas y epidemiológicas en niños con infecciones respiratorias
}

\author{
Juan C. Flores C., Cecilia Vizcaya A., Rafael Araos B., Luisa Montecinos P., Paula Godoy M., \\ Fernando Valiente-Echeverría, Cecilia Perret P., Patricia Valenzuela C., Tamara Hirsch B. y Marcela Ferrés G.
}

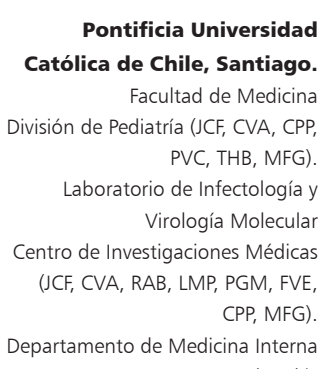

$(\mathrm{RAB})^{*}$

Conflictos de interés ninguno

Fuentes de financiamiento: Los autores agradecen a Laboratorio Roche por proveer Light Cycler reaction kit.

*Actualmente en Universidad del Desarrollo, Departamento de Infectología.

Recibido: 14 de junio de 2011 Aceptado: 30 de agosto de 2011

Correspondencia a: Marcela Ferrés G. mferres@med.puc.cl

\section{Introducción}

E n el año 2005, Allander y cols., describieron un nuevo parvovirus humano procedente de muestras de niños con infecciones respiratorias agudas (IRA). Fue llamado bocavirus humano (HBoV), perteneciente al género Bocavirus, y a la familia Parvoviridae ${ }^{1}$. Desde su descubrimiento se han descrito cuatro grupos o "clades" (HBoV 1-4), asociados con infecciones respiratorias y gastrointestinales en países de todo el mundo ${ }^{2-4}$.

Diversos estudios han reportado la presencia de $\mathrm{HBoV}$ en el tracto respiratorio, utilizando técnicas de biología molecular, con frecuencias que varían entre 1,5 y $19 \%$ entre niños con IRA ${ }^{5-15}$, y entre 0 y $43 \%$ en muestras respiratorias de pacientes asintomáticos ${ }^{7,14,16-20}$. Por otra parte, se ha descrito co-infección con otros agentes virales, principalmente virus respiratorio sincicial (VRS) y adenovirus, entre 9 y $90 \%{ }^{13,16}$. Otros estudios han sugerido la excreción asintomática de $\mathrm{HBoV}$ en pacientes bajo un año de edad $(8,6 \%)$, que puede prolongarse hasta por seis meses ${ }^{20,21}$.

Con el objetivo de describir el perfil clínico y epidemiológico de este nuevo virus en pacientes bajo 5 años de edad en Chile, diseñamos un estudio para evaluar la presencia de $\mathrm{HBoV}$ en pacientes que consultaron por IRA, y en niños asintomáticos. Comparamos dos cohortes de pacientes: aquellos con muestras positivas para $\mathrm{HBoV}$ como único agente identificado, y los que tenían una coinfección con otro virus respiratorio. Además evaluamos su excreción viral en un grupo de niños asintomáticos a través de reacción de polimerasa en cadena (RPC) y realizamos análisis filogenético de las cepas chilenas para compararlas con las descritas en otros países.

\section{Material y Métodos}

Diseño del estudio. Estudio de cohorte no concurrente, aprobado por el Comité de Ética de la Dirección de Investigación, Pontificia Universidad Católica (PUC) de Chile.

Pacientes sintomáticos. Los pacientes se reclutaron desde dos fuentes. Entre marzo y noviembre de 2007, se enrolaron niños bajo 5 años de edad que consultaron por IRA en centros ambulatorios donde se les tomó una muestra de hisopado nasofaríngeo (HNF) como parte de 
un programa de vigilancia activa de virus respiratorios circulantes en Santiago, Chile. En segundo lugar, entre mayo y agosto de 2007 se incluyeron niños bajo 5 años de edad con IRA hospitalizados, o que consultaron en el Servicio de Urgencia o centros ambulatorios de nuestra institución, cuyas muestras fueron recibidas en el Laboratorio de Infectología y Virología Molecular para diagnóstico etiológico.

Grupo control. Los niños asintomáticos se reclutaron entre pacientes que acudieron a control sano habitual o que ingresaron al hospital para cirugía electiva u otro procedimiento. Los criterios de inclusión fueron: ausencia de síntomas respiratorios en los 10 días previos y edad menor a 5 años. Si el resultado de la RPC para $\mathrm{HBoV}$ era positivo, se realizó un seguimiento telefónico para evaluar aparición de síntomas respiratorios dentro de 7 días posteriores a la toma de muestra.

Características biodemográficas. Se registraron variables clínicas y demográficas en una base de datos, incluyendo: edad, sexo, fecha de obtención de HNF, síntomas, diagnóstico, patrón radiológico, ingreso al hospital, requerimiento de oxígeno y tratamiento recibido. Se revisó la ficha clínica y se contactó a los pacientes por teléfono para realizar su seguimiento. Se compararon los resultados entre los pacientes que tenían $\mathrm{HBoV}$ como único agente identificado, con aquellos en que se demostró co-infección con otro virus respiratorio.

Definiciones clínicas. IRA fueron definidas como cualquiera de los siguientes diagnósticos: infección respiratoria alta (presencia de rinorrea y/o tos, con o sin fiebre, y ausencia de dificultad respiratoria y signos de neumonía u obstrucción de vía aérea), bronquiolitis (primer o segundo episodio de sibilancias en lactantes bajo 2 años de edad, con signos y síntomas de infección viral y dificultad respiratoria), sibilancias recurrentes (tres o más episodios de sibilancias), o neumonía (infección respiratoria con imágenes de relleno alveolar en la radiografía de tórax).

Muestras clínicas y métodos de diagnóstico virológico. Las muestras respiratorias se obtuvieron utilizando tórulas de dacrón para $\mathrm{HNF}$, y trasladadas el mismo día al laboratorio a una temperatura de $4^{\circ} \mathrm{C}$ en medio de transporte viral. Las muestras se estudiaron utilizando técnicas de inmunofluorescencia directa (IFD) para virus respiratorio sincicial (VRS), adenovirus (ADV), virus influenza (Flu) A y B, parainfluenza (paraFlu) 1, 2, 3 y metapneumovirus humano (MNVh) (D3 UltraTM DFA Respiratory Virus and MPV. DHI, California, USA)®. Las muestras negativas para MNVh por IFD se estudiaron además mediante técnica de RPC con transcriptasa reversa según la técnica descrita por Mackay ${ }^{22}$. Todas las muestras fueron procesadas en el Laboratorio de Infectología y Virología Molecular de la Facultad de Medicina, PUC de Chile.

Técnica de RPC. El ácido nucleico se extrajo desde las muestras utilizando High Pure Viral Nucleic Acid Kit (Roche) y siguiendo las instrucciones del fabricante. El genoma viral se estudió siguiendo el protocolo descrito por Xiaoyan Lu, 2006 $6^{23}$, utilizando partidores del gen $n s 1$ (290 pb) (forward: 5'-TAT GGC CAA GGC AAT CGT CCA AG-3', reverse: 5'-GCC GCG TGA ACA TGA GAA ACA GA-3').

RPC convencional. La RPC se realizó en una mezcla de 12,5 $\mu$ L que contenía: $2,5 \mu$ l de tampón de reacción $5 \mathrm{x}$ (Flexi Taq, Promega), 2,5 $\mu \mathrm{l}$ de $\mathrm{MgCl}_{2}(25 \mathrm{mM}), 0,5 \mu \mathrm{l}$ de dNTP $(10 \mathrm{mM}), 0,75 \mu \mathrm{l}$ de partidor forward $(10 \mu \mathrm{M})$, $0,75 \mu \mathrm{l}$ de partidor reverse para el gen $n s 1(10 \mu \mathrm{M})$, Taq polimerasa (Go Taq Flexi, Promega) 2,5 unidades/ $\mu 1,5$ $\mu l$ de ADN, y agua libre de nucleasa. Después de preparar la mezcla, se realizó un paso de denaturación de $5 \mathrm{~min}$ a $94^{\circ} \mathrm{C}$, seguido de 35 ciclos de amplificación $\left(94^{\circ} \mathrm{C}\right.$ por $30 \mathrm{~s}, 65^{\circ} \mathrm{C}$ por $30 \mathrm{~s}, 72^{\circ} \mathrm{C}$ por $30 \mathrm{~s} \mathrm{y} 7 \mathrm{~min}$ de extensión final a $72^{\circ} \mathrm{C}$ ), en el equipo 2720 Thermal Cycler (Applied Biosystems). El fragmento amplificado de $290 \mathrm{pb}$ se visualizó en un gel de agarosa al 2\%. El primer fragmento amplificado de $\mathrm{HBoV}$ se secuenció en el Laboratorio de Diversidad Molecular, PUC de Chile, y fue comparado con las secuencias publicadas en GenBank para confirmación. Luego se utilizó como control positivo para el estudio. La identidad genética resultó ser de 99\% para el gen ns1 de HBoV (número de acceso: DQ988934-2).

RPC en tiempo real. HBoV se estudió en el grupo de niños asintomáticos mediante RPC en tiempo real buscando el gen ns1, como se describe anteriormente, utilizando el equipo Light Cycler ${ }^{\circledR}$ Fast Start DNA Master Syber Green I Kit (Roche). La mezcla de $10 \mu \mathrm{L}$ contenía: 6,2 $\mu 1$ de agua libre de nucleasa, $0,8 \mu 1$ de $\mathrm{MgCl}_{2}$ (25 mM), $0,5 \mu \mathrm{l}$ de partidor forward $(10 \mu \mathrm{M}), 0,5 \mu \mathrm{l}$ de partidor reverse para el gen $n s 1(10 \mu \mathrm{M}), 1 \mu \mathrm{l}$ de LC Fast start reaction mix, y $1 \mu \mathrm{l}$ de ADN. La amplificación se realizó utilizando el equipo Light Cycler (Roche) siguiendo los siguientes pasos: $95^{\circ} \mathrm{C}$ de preincubación por $10 \mathrm{~s} ; 32$ ciclos que consistían en: $95^{\circ} \mathrm{C}$ por $10 \mathrm{~s}, 63^{\circ} \mathrm{C}$ por $5 \mathrm{~s}, \mathrm{y} 72^{\circ} \mathrm{C}$ por $12 \mathrm{~s}$. Curva de melting $95^{\circ} \mathrm{C}$ por $0 \mathrm{~s}, 65^{\circ} \mathrm{C}$ por $15 \mathrm{~s} \mathrm{y}$ $95^{\circ} \mathrm{C}$ por $0 \mathrm{~s}$. La temperatura de melting para el control positivo fue $85^{\circ} \mathrm{C}$, considerando como positivos valores de $85 \pm 1{ }^{\circ} \mathrm{C}$. Se utilizaron precauciones estándares en la extracción de las muestras y en la realización de la RPC para evitar contaminación.

Análisis filogenético. Para realizar el análisis filogenético, se amplificaron fragmentos de los genes ns1 y vp1 


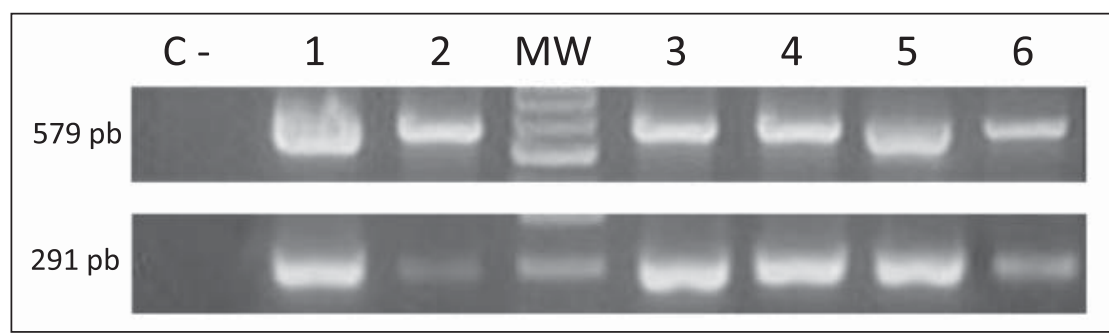

Figura 1. Arriba, productos de RPC amplificando gen vp1 de HBoV obtenido de seis muestras de niños chilenos. Los resultados positivos se indican por una banda de 579 pb (líneas 1 a 6). Abajo, productos de RPC amplificando gen ns1 de HBoV obtenido de seis muestras de niños chilenos. Los resultados positivos se indican por una banda de 291 pb (líneas 1 a 6). Línea C-: control negativo; línea MW: Ladder de ADNA de $1 \mathrm{~Kb}$.

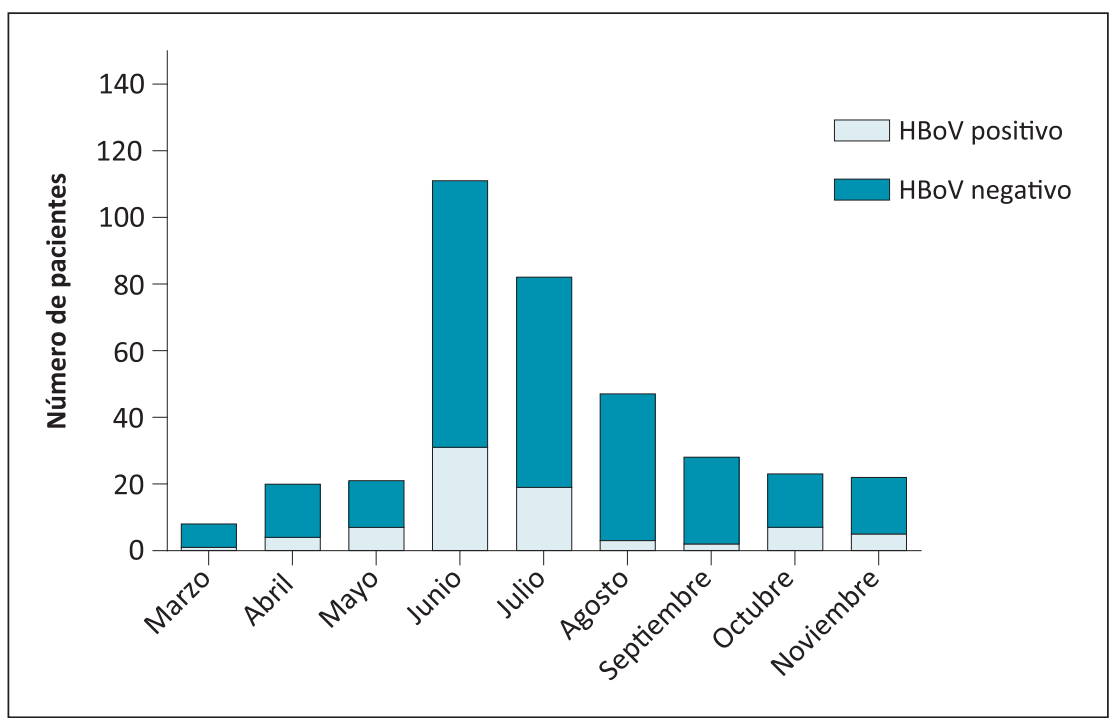

Figura 2. Distribución estacional de 79 muestras positivas para HBoV en niños bajo 5 años de edad, Santiago, Chile, 2007.
Los números de acceso a GenBank para las secuencias de $\mathrm{HBoV}$ utilizadas en la construcción del árbol filogenético fueron las siguientes: CU74W EU262979; CU8N EU262978, Bovine parvovirus-1 strain Abinanti BPV DQ335247; HK20 EF450736; HK10 EF450726; HK1 EF450717; CU6 EF203920; WLL-3 EF584447; WLL-2 EF441262; BJ3722 DQ988934; CU74 EF203922; CU49 EF203921; BJ3064 DQ988933; WLL-1 DQ778300; CRD2 DQ340570; st2 DQ000496; st1 DQ000495; Minute virus of canines strain: HM-6 MCV AB158475.

Análisis estadístico. Las variables continuas se presentan como mediana y rango, y las variables categóricas como frecuencia y porcentaje.

Las características clínicas se compararon utilizando el test exacto de Fisher y el test $U$ Mann-Whitney.

Se consideró un valor $\mathrm{p}<0,05$ como estadísticamente significativo.

El análisis estadístico se realizó utilizando el programa Statistical Package for the Social Sciences (SPSS), versión 15.0.

\section{Resultados}

Detección viral. Se detectó $\mathrm{HBoV}$ en 79 muestras $(21,8 \%)$ de un total de $362 \mathrm{HNF}$ obtenidos en niños bajo 5 años de edad con IRA. En 24\% (19/79) de las muestras positivas, $\mathrm{HBoV}$ fue el único agente identificado, y en $76 \%(60 / 79)$ se detectó co-infección con otro agente viral: VRS en $65 \%(39 / 60)$, paraFlu en $13,3 \%(8 / 60)$, MNVh en $13,3 \%(8 / 60)$, ADV en $8,3 \%(5 / 60)$ y virus Flu B en $3,3 \%(2 / 60)$.

Distribución estacional. Los casos positivos para $\mathrm{HBoV}$ se detectaron entre los meses de marzo y noviembre. La mayoría $(63,3 \%)$ se concentró en el período de invierno del hemisferio sur (junio y julio) (Figura 2).

de seis pacientes chilenos de acuerdo al protocolo descrito por Sloots en el año $2006^{12}$. Los fragmentos amplificados se analizaron mediante electroforesis en gel de agarosa al $2 \%$, y purificados para posterior secuenciación del ADN (Figura 1). Los fragmentos VP1 y NS1, de 579 pb y 291 $\mathrm{pb}$ respectivamente, se secuenciaron (Macrogen Inc, Bethesda, Maryland, USA) y se analizaron para obtener relaciones filogenéticas. Se realizó un alineamiento múltiple de secuencias con el programa Clustal $\mathrm{W}^{24}$. Los árboles filogenéticos basados en la secuencia de los genes ns1 y vp1 se construyeron por el método del vecino más próximo (Neighbor-Joining), y la matriz de distancia se calculó a través del método Kimura (two-parameters) ${ }^{25}$. La estabilidad se estimó por análisis de confianza (bootstrap) de 1.000 replicaciones. El cálculo de distancias matrices y la construcción de los árboles filogenéticos se realizaron utilizando el software MEGA 4.025.
Características clínicas y demográficas. Se describen en 62 pacientes, sobre un total de 79 que resultaron ser positivos para HBoV. Los síntomas más frecuentes fueron tos $(97,9 \%)$, fiebre $(89,7 \%)$ y rinorrea $(89,7 \%)$. Se constató diarrea en $26,5 \%$ de los pacientes positivos para $\mathrm{HBoV}$. Bronquiolitis y sibilancias recurrentes fueron los diagnósticos más frecuentes, consignados en más de $50 \%$ de los casos. El antecedente de asma o sibilancias recurrentes estaba presente en $32,7 \%$ de los pacientes. El patrón radiográfico más frecuente fue compromiso intersticial, en 43,4\% (10/23) del total de casos en quienes se consideró necesaria la evaluación con radiografía de tórax. Al comparar los pacientes con $\mathrm{HBoV}$ como único agente identificado con aquellos con co-infección, este último grupo mostró mayor gravedad en la presentación clínica, con una diferencia estadísticamente significativa 
en tres variables analizadas: dificultad respiratoria, requerimiento de oxígeno y hospitalización (Tabla 1). Los pacientes positivos para HBoV tenían bajo 2 años en $69,6 \%$ de los casos (Figura 3). Además se observa que a mayor edad, existe una disminución progresiva de los pacientes positivos para $\mathrm{HBoV}$.

Grupo control. Se analizaron 16 muestras obtenidas en niños asintomáticos, utilizando RPC convencional y en tiempo real. Se detectó HBoV en $3(18,8 \%)$ y 6 (37,5\%) muestras de HNF, respectivamente. Durante el seguimiento, un paciente que resultó positivo por ambas técnicas, presentó síntomas respiratorios (laringitis aguda) antes de siete días desde la toma de muestra.

Análisis filogenético. Los fragmentos amplificados de los genes ns1 y vp1 de HBoV obtenidos desde muestras chilenas se secuenciaron según se detalla en la sección Materiales y Métodos. El análisis filogenético de las secuencias obtenidas se muestra en la Figura 4. Las secuencias nucleotídicas de los virus obtenidos desde muestras chilenas están fuertemente relacionadas con los genotipos st1 y st2 descritos por Allander en Suecia ${ }^{1}$. La mayoría de los polimorfismos identificados en este estudio no se tradujeron en cambios a nivel aminoacídico de los productos génicos correspondientes.

\section{Discusión}

Nuestro estudio encontró presencia de genoma viral de $\mathrm{HBoV}$ en $21,8 \%$ de las muestras de HNF obtenidas en pacientes bajo de edad 5 años que consultaron por IRA, y en $18,8 \%$ en niños asintomáticos, usando RPC convencional. Se detectó co-infección en $76 \%$ de las muestras positivas para HBoV. Este grupo mostró una presentación clínica más grave al compararlo con los pacientes en quienes se identificó $\mathrm{HBoV}$ como agente único, de los cuales ninguno requirió ingreso al hospital.

La prevalencia real de $\mathrm{HBoV}$ en pacientes con IRA utilizando técnicas de biología molecular probablemente está subestimada, considerando la mayor sensibilidad que ha demostrado tener la RPC en tiempo real. Esto fue observado en nuestro estudio al comparar las muestras positivas entre las dos técnicas en el grupo de asintomáticos $(37,5$ vs $18,8 \%)$.

Desde que HBoV fue descrito por Allander y cols. en 2005, se han publicado varios artículos con información epidemiológica y virológica realizados en países de América, Europa, Asia, Oceanía y Africa $^{5-15}$. Nuestro estudio reporta una prevalencia más alta en niños con IRA $(21,8 \%)$, al compararla con los datos reportados en la literatura: de $1,5 \%$ a $19 \%{ }^{5-15}$. Probablemente esta diferencia se explica porque nuestras muestras fueron
Tabla 1. Características clínicas y demográficas de 62 pacientes chilenos con infección por HBoV

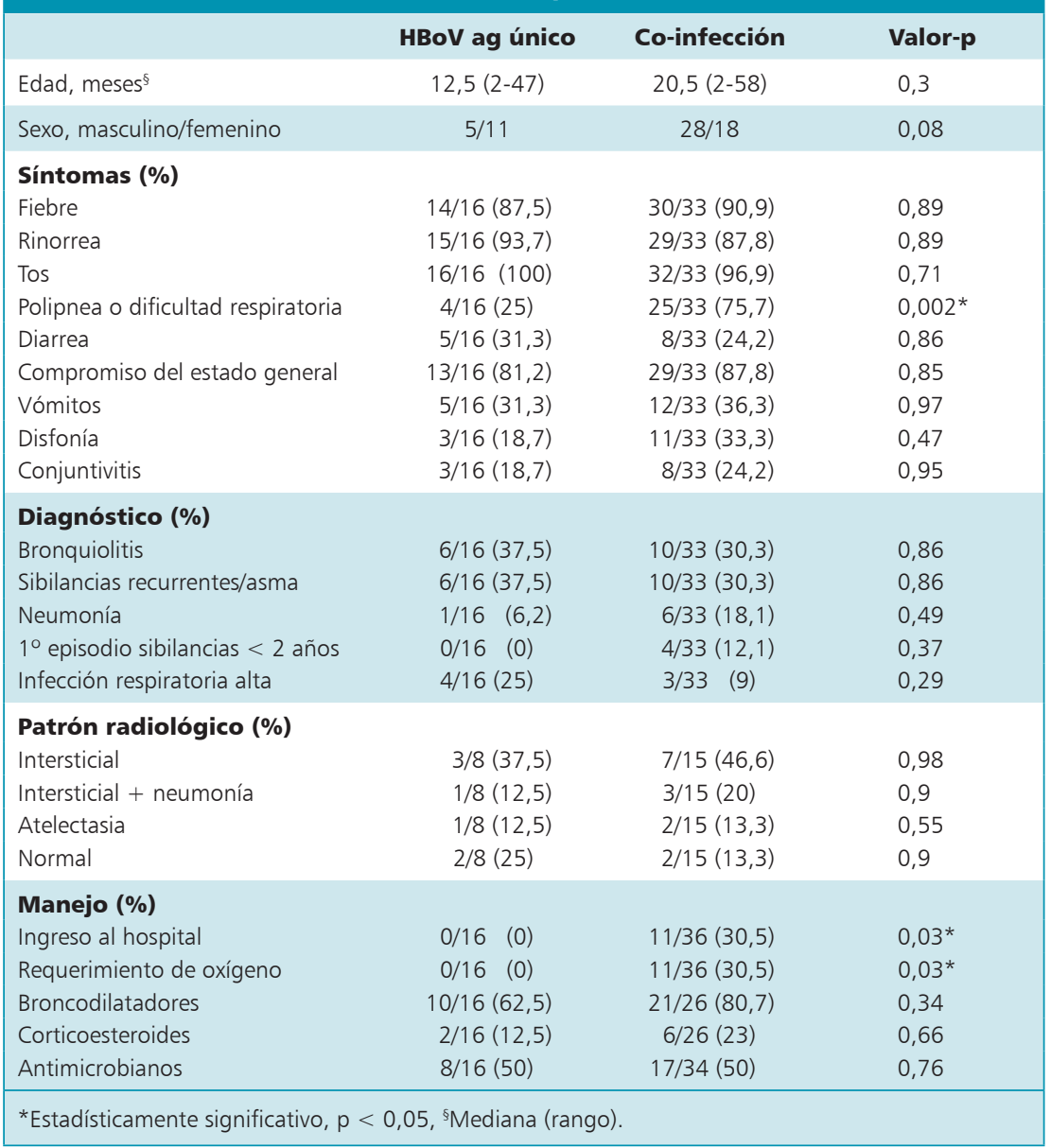

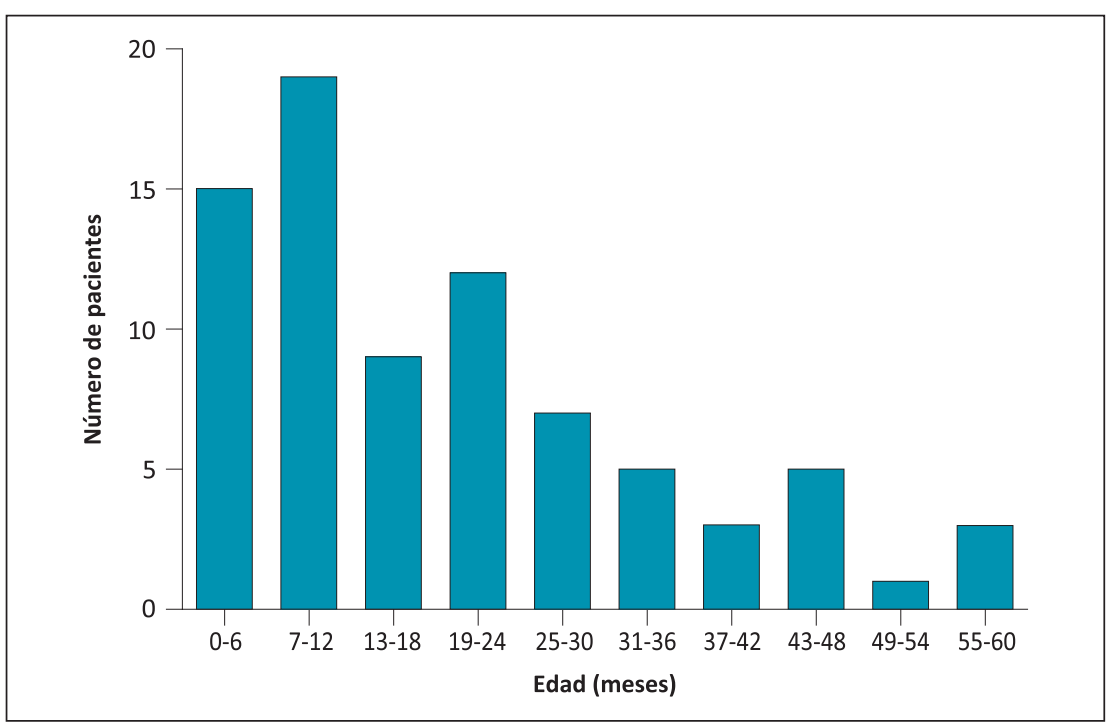

Figura 3. Distribución por edad de 79 pacientes con muestras positivas para HBoV en pacientes bajo 5 años de edad, Santiago,Chile, 2007. 
Figura 4. Análisis Filogenético. Arboles filogenéticos construidos en base al método del vecino más próximo (Neighbor-Joining) utilizando la secuencia nucleotídica (panel izquierdo) y la secuencia de aminoacídica (panel derecho) de los genes ns1 (A), vp1 (B) y sus productos génicos, respectivamente. Los valores de confianza (bootstrap) se indican en cada rama; st1 y st2: cepas originales descritas por Allander ${ }^{1}$; PedCHI: cepas HBoV de niños chilenos. Además de señala la fecha de la obtención de la muestra para cada cepa. Escala en el extremo inferior izquierdo indica 0,05 sustituciones por nucleótido.

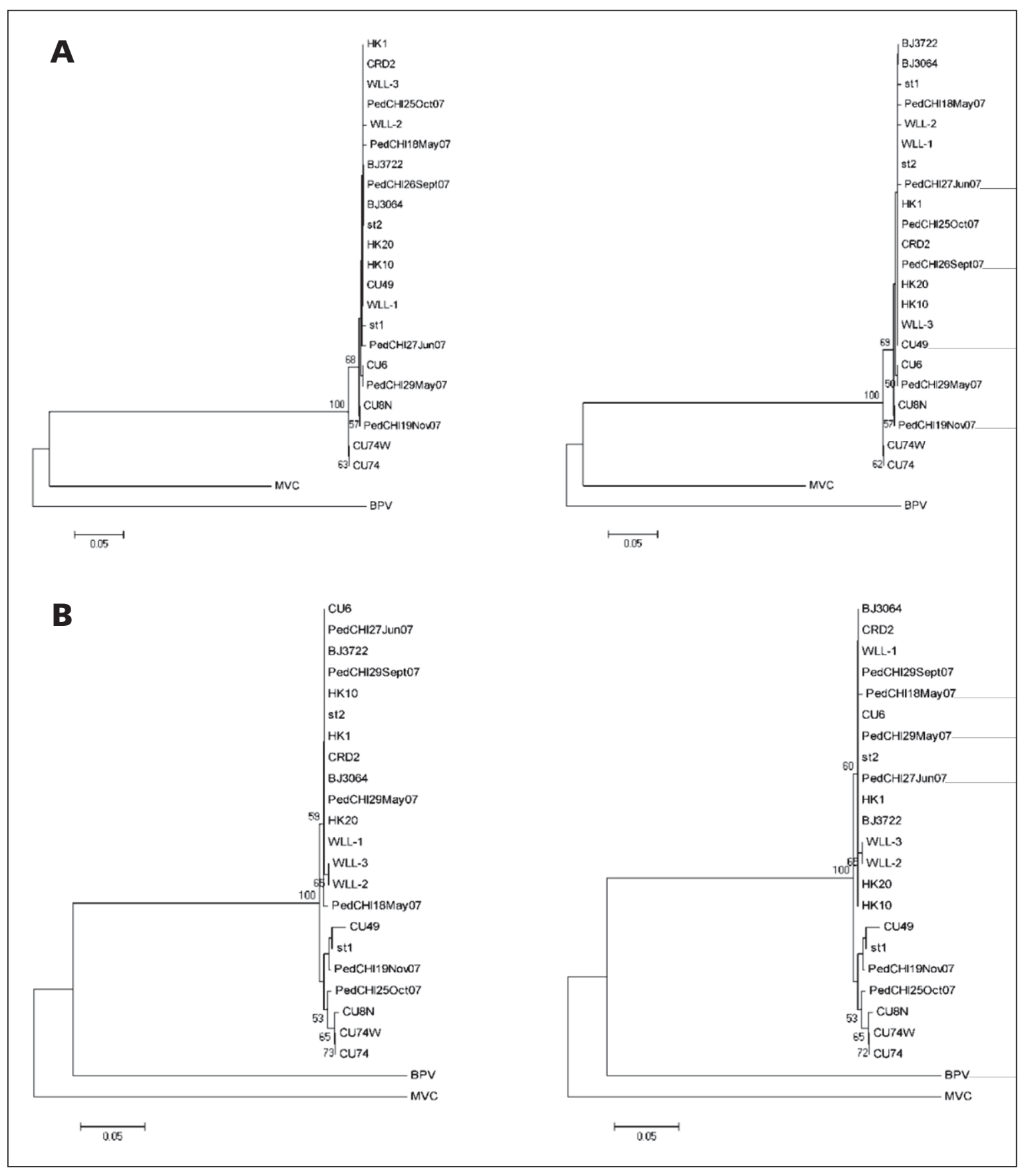

obtenidas en su mayoría en período de invierno, donde la circulación de virus respiratorios es mayor. La distribución por edad de los pacientes positivos para $\mathrm{HBoV}$ fue similar a lo reportado: principalmente niños entre 6 meses y 2 años de edad ${ }^{1,7,8,9,11,14,16,17}$. La presentación clínica tampoco fue diferente a lo ya descrito: tos, fiebre y rinorrea fueron los síntomas más frecuentes encontrados en nuestros pacientes, y además $25 \%$ tuvo diarrea. El rol de $\mathrm{HBoV}$ como causa de crisis asmáticas también ha sido sugerido por algunos trabajos ${ }^{26-28}$. El antecedente de asma o sibilancias recurrentes estaba presente en alrededor de $1 / 3$ de nuestros pacientes, tanto en los que tenían $\mathrm{HBoV}$ como agente único, como en los co-infectados con virus que clásicamente se presentan con sibilancias (VRS). La principal diferencia entre ambos grupos fue la gravedad de los síntomas. 
El rol de HBoV en IRA ha sido cuestionado por varios motivos. Primero, la mayoría de los estudios han usado técnicas diagnósticas basadas en biología molecular (RPC). Un resultado positivo en este contexto puede significar infección aguda o excreción viral prolongada de partículas virales, vivas o inactivadas ${ }^{28}$. Un gran número de pacientes se presentan con cargas virales bajas ${ }^{14,23,29,30}$, y ha sido demostrada su excreción hasta por 6 meses $^{20,21}$, lo cual sugiere persistencia del virus por períodos prolongados. Además, varios estudios han reportado alta prevalencia de $\mathrm{HBoV}$ en pacientes asintomáticos, entre 5 y $43 \%{ }^{18-20}$, altas tasas de co-infección con otros virus, y su presencia en deposiciones ${ }^{31-34}$, suero $^{9,14}$ y orina $^{13}$, lo cual sugiere una infección sistémica.

En nuestro estudio encontramos una diferencia estadísticamente significativa entre el grupo en que $\mathrm{HBoV}$ fue agente único, en comparación al grupo con co-infección (principalmente VRS), en tres variables que reflejan mayor gravedad: dificultad respiratoria, hospitalización y requerimiento de oxígeno. Esto podría sugerir que $\mathrm{HBoV}$ como patógeno respiratorio tendría una repercusión clínica limitada, cuestionando su rol patogénico en IRA.

Por otra parte, nueva evidencia apoya el rol de $\mathrm{HBoV}$ como patógeno respiratorio en niños. Allander y cols. ${ }^{14}$, sugiere una teoría en que cargas virales altas de $\mathrm{HBoV}$ estarían asociadas con síntomas respiratorios y viremia, indicando infección sistémica, mientras que cargas virales bajas en secreciones respiratorias podrían indicar excreción asintomática. El estudio de Kantola y cols. ${ }^{35}$, encontró viremia y altas cargas virales en secreciones nasofaríngeas en un subgrupo de pacientes con sibilancias y ausencia de otros virus respiratorios. Además todos ellos mostraron evidencia serológica de infección aguda.

Pocos estudios han evaluado la respuesta inmune a $\mathrm{HBoV}$, principalmente por la dificultad en obtener antígenos virales recombinantes y métodos de diagnóstico estandarizados ${ }^{35-39}$. Un inmunoensayo basado en la proteína 2 (VP2) fue desarrollado por Söderlund-Venermo y cols. ${ }^{40}$. El examen mostró una sensibilidad de $97 \%$ y especificidad de $99,5 \%$. En este estudio, un total de 48 (19\%) de 258 niños con sibilancias tenían evidencia serológica de infección primaria por $\mathrm{HBoV}$. Otro estudio reciente realizado por Don y cols. ${ }^{41}$, utilizó el mismo método para estudiar a 101 niños ingresados por neumonía. Se encontró evidencia serológica de infección aguda en $12 \%$ de los pacientes. Además, la seropositividad a $\mathrm{HBoV}$ se incrementó con la edad, alcanzando casi $100 \%$ en adultos $^{40,41}$. La presencia de IgM específica para $\mathrm{HBoV}$, además de un incremento en la IgG ha sido descrito como un método sensible para diagnóstico de infecciones agudas y ha aportado evidencia importante en aclarar el rol patogénico de este agente en IRA. La serología es usada en forma rutinaria para diagnóstico de infecciones por parvovirus B19, y en forma similar, debería llegar a ser el examen de elección para diagnóstico de $\mathrm{HBoV}^{38,42}$.

La importante similitud entre cepas chilenas y las descritas originalmente en el hemisferio norte, basado en el análisis filogenético, sugiere que $\mathrm{HBoV}$ es un virus altamente conservado, y exámenes serológicos deberían tener aplicación e interpretación universal en todo el mundo.

Nuestro estudio tiene limitaciones que se deben considerar en el análisis. Considerando la evidencia actual, la RPC cualitativa no es el método de elección para describir y estudiar un virus que se excreta en forma asintomática por períodos prolongados ${ }^{20,21}$. Algunos datos fueron obtenidos en forma retrospectiva, lo cual podría tener influencia en los resultados ("recall bias" o error de memoria), y se reclutaron pocos niños asintomáticos.

En conclusión, HBoV está presente en población chilena, y cuando se detecta otro agente viral en la muestra respiratoria, se evidencia una expresión clínica de mayor gravedad. La evidencia actual ha demostrado excreción viral por períodos prolongados, lo cual podría explicar su presencia en niños asintomáticos. Esto a su vez hace que las técnicas cualitativas de biología molecular no sean la técnica más adecuada para estudiar la epidemiología y realizar diagnóstico de infección aguda. En este ámbito, la serología se perfila como el método de elección.

Agradecimientos. Los autores agradecen a todos los niños y padres que participaron en este estudio, al laboratorio Roche por proveer de Light Cycler reaction kit, a los estudiantes de medicina Alex Arenas, Javiera Pardo y Daniela Gutiérrez por su ayuda en la recolección de los datos, y a los doctores José Antonio Castro-Rodríguez, Marcelo López y Paul Harris por su gentil revisión de este manuscrito.

\section{Resumen}

Introducción: Bocavirus humano (HBoV) es un nuevo parvovirus encontrado en niños con infecciones respiratorias agudas (IRA). Objetivos: Describir la epidemiología y perfil clínico en niños $\leq 5$ años con IRA, comparando aquellos con $\mathrm{HBoV}$ como único agente identificado, con los que tenían co-infección con otro virus respiratorio. Además se evaluó su prevalencia en niños asintomáticos, y se realizó análisis filogenético. Materiales y Métodos: Se investigó la presencia de $\mathrm{HBoV}$, por medio de reacción de polimerasa en cadena, en muestras de secreción nasofaríngea obtenida en niños con IRA y en controles asintomáticos, entre 2007 y 2008. Resultados: Se detectó HBoV en 79 (21,8\%) de 362 muestras obtenidas en pacientes con IRA. En 60/79 $(76 \%)$, se demostró co-infección. Los síntomas más frecuentes fueron tos, fiebre y rinorrea. Los pacientes con $\mathrm{HBoV}$ como único agente identificado mostraron frecuencias significativamente menores de dificultad 
respiratoria, requerimiento de oxígeno y hospitalización, comparado con los co-infectados. HBoV se detectó en 6/16 (37,5\%) muestras de niños asintomáticos. El análisis filogenético de las cepas chilenas demuestra estrecha relación con las cepas originales. Conclusiones: $\mathrm{HBoV}$ está presente en niños chilenos con IRA y asintomáticos. La gravedad de la enfermedad fue mayor en el grupo con co-infección.

\section{Referencias}

1.- Allander T, Tammi M T, Eriksson M, Bjerkner A, Tiveljung-Lindell A, Andersson B. Cloning of a human parvovirus by molecular screening of respiratory tract samples. Proc Natl Acad Sci USA 2005; 102: 12891-6.

2.- Chieochansin T, Kapoor A, Delwart E, Poovorawan Y, Simmonds P. Absence of detectable replication of human bocavirus species in respiratory tract. Emerg Infect Dis 2009; 15: 1503-5.

3.- Song J R, Jin Y, Xie Z P, Gao H C, Xiao N G, Chen W X, et al. Novel human bocavirus in children with acute respiratory tract infection. Emerg Infect Dis 2010; 16: 324-7.

4.- Kapoor A, Simmonds P, Slikas E, Li L, Bodhidatta L, Sethabutr O, et al. Human bocaviruses are highly diverse, dispersed, recombination prone, and prevalent in enteric infections. J Infect Dis 2010; 201: 1633-43.

5.- Bastien N, Brandt K, Dust K, Ward D, Li Y. Human bocavirus infection, Canada. Emerg Infect Dis 2006; 12: 848-50.

6.- Arnold J C, Singh K K, Spector S A, Sawyer M H. Human bocavirus: prevalence and clinical spectrum at a children hospital. Clin Infect Dis 2006; 43: 283-8.

7.- Kesebir D, Vázquez M, Weibel C, Shapiro E D, Ferguson D, Landry M L, et al. Human bocavirus infection in young children in the United States: Molecular epidemiological profile and clinical characteristics of a newly emerging respiratory virus. J Infect Dis 2006; 194: 1276-82.

8.- Ma X, Endo R, Ishiguro N, Ebihara T, Ishiko H, Ariga T, et al. Detection of human bocavirus in Japanese children with lower respiratory tract infections. J Clin Microbiol 2006; 44: 1132-4.

9.- Qu X W, Duan Z J, Qi Z Y, Xie Z P, Gao H C, Liu W P, et al. Human bocavirus infection, People's Republic of China. Emerg Infect Dis 2007; 13: 165-8.

10.- Choi E H, Lee H J, Kim S J, Eun B W, Kim N H, Lee JA, et al. The association of newly identified respiratory viruses with lower respiratory tract infections in Korean children, 2000-2005. Clin Infect Dis 2006; 43: 585-92.

11.- Manning A, Russel V, Eastick K, Leadbetter G H, Hallam N, Templeton K, et al. Epidemiological profile and clinical associations of human bocavirus and other human parvoviruses. J Infect Dis 2006; 194: 1283-90.

12.- Sloots T P, McErlean P, Speicher D J, Arden K E, Nissen M D, Mackay I M. Evidence of human coronavirus HKU1 and human bocavirus in Australian children. J Clin Virol 2006; 35: 99-102.

13.- Pozo F, García-García ML, Calvo C, Cuesta I, Pérez-Breña P, Casas I. High incidence of human bocavirus infection in children in Spain. J Clin Virol 2007; 40: 224-8.

14.- Allander T, Jartti T, Gupta S, Niesters H G, Lehtinen P, Osterback R, et al. Human bocavirus and acute wheezing in children. Clin Infect Dis 2007; 44: 904-10.

15.- Weissbrich B, Neske F, Schubert J, Tollmann F, Blath K, Blessing K, et al. Frequent detection of bocavirus DNA in German children with respiratory tract infections. BMC Infect Dis 2006; 6: 109.

16.- Fry A M, Lu X, Chittaganpitch M, Peret T, Fischer J, Dowell S F, et al. Human bocavirus: a novel parvovirus epidemiologically associated with pneumonia requiring hospitalization in Thailand. J Infect Dis 2007; 195: 1038-45.

17.- Maggi F, Andreoli E, Pifferi M, Meschi S, Rocchi J, Bendinelli M. Human bocavirus in Italian patients with respiratory disease. J Clin Virol 2007; 38: 321-5.

18.- Longtin J, Bastien M, Gilca R, Leblanc E, de Serres G, Bergeron M G, et al. Human bocavirus infections in hospitalized children and adults. Emerg Infect Dis 2008; 14: 217-21.

19.- García-García M L, Calvo C, Pozo F, PérezBreña P, Quevedo S, Bracamonte T, et al. Human bocavirus detection in nasopharyngeal aspirates of children without clinical symptoms of respiratory infection. Pediatr Infect Dis J 2008; 27: 358-60.

20.- von Linstow M L, Høgh M, Høgh B. Clinical and epidemiologic characteristics of human bocavirus in Danish infants: results from a prospective birth cohort study. Pediatr Infect Dis J 2008; 27: 897-902.

21.- Brieu N, Guyon G, Rodière M, Segondy M, Foulongne V. Human bocavirus infection in children with respiratory tract disease. Pediatr Infect Dis J 2008; 27: 969-73.

22.- Mackay I M, Jacob K C, Woolhouse D, Waller K, Syrmis M W, Whiley D M, et al. Molecular assays for detection of human metapneumovirus. J Clin Microbiol 2003; 41: 100-5.

23.- Lu X, Chittaganpitch M, Olsen S J, Mackay I M, Sloots T P, Fry A M, et al Real-time PCR assays for detection of bocavirus in human specimens. J Clin Microbiol 2006; 44: 3231-5.

24.- Thompson J D, Higgins D G, Gibson T J. Clustal W. Improving the sensitivity of progressive multiple sequence alignment through sequence weighting, position-specific gap penalties and weight matrix choice. Nucleic Acids Res 1994; 22: 4673-80.

25.- Tamura K, Dudley J, Nei M, Kumar S. MEGA4: Molecular Evolutionary Genetics Analysis (MEGA) software version 4.0. Mol Biol Evol 2007; 24: 1596-9.

26.- Calvo C, García-García M L, Pozo F, Carvajal O, Pérez-Breña P, Casas I. Clinical characteristics of human bocavirus infections compared with other respiratory viruses in Spanish children. Pediatr Infect Dis J 2008; 27 : 677-80.

27.- Maffey A F, Barrero P R, Venialgo C, Fernández F, Fuse V A, Saia M, et al. Viruses and atypical bacteria associated with asthma exacerbations in hospitalized children. Pediatr Pulmonol 2010; 45: 619-25.

28.- Arnold J C. Human bocavirus in children. Pediatr Infect Dis J 2010; 29: 557-8.

29.- Foulongne V, Rodière M, Segondy M. Human bocavirus in children. Emerg Infect Dis 2006; 12: 862-3.

30.- Lin F, Zeng A, Yang N, Lin H, Yang E, Wang $\mathrm{S}$, et al. Quantification of human bocavirus in lower respiratory tract infections in China. Infect Agent Cancer 2007; 2: 3 .

31.- Lau S K, Yip CC, Que T L, Lee R A, Au-Yeung R K, Zhou B, et al. Clinical and molecular epidemiology of human bocavirus in respiratory and fecal samples from children in Hong Kong. J Infect Dis 2007; 196: 986-93.

32.- Lee J I, Chung J Y, Han T H, Song M O, Hwang E S. Detection of human bocavirus in children hospitalized because of acute gastroenteritis. J Infect Dis 2007; 196: 994-7.

33.- Vicente D, Cilla G, Montes M, Pérez-Yarza EG, Pérez-Trallero E. Human bocavirus, a respiratory and enteric virus. Emerg Infect Dis 2007; 13: 636-7.

34.- Albuquerque M C, Rocha L N, Benati F J, 
Soares C C, Maranhão A G, Ramírez M L, et al. Human bocavirus infection in children with gastroenteritis, Brazil. Emerg Infect Dis 2007; 13: 1756-8.

35.- Kantola K, Hedman L, Allander T, Jartti T, Lehtinen P, Ruuskanen O, et al. Serodiagnosis of human bocavirus infection. Clin Infect Dis 2008; 46: 540-6.

36.- Endo R, Ishiguro N, Kikuta H, Teramoto S, Shirkoohi R, Ma X, et al. Seroepidemiology of human bocavirus in Hokkaido Prefecture, Japan. J Clin Microbiol 2007; 45: 3218-23.
37.- Kahn J S, Kesebir D, Cotmore S F, D’Abramo A $\mathrm{Jr}$, Cosby C, Weibel C, et al. Seroepidemiology of human bocavirus defined using recombinant virus-like particles. J Infect Dis 2008; 198: 41-50.

38.- Simmonds P. Steps towards serological diagnosis of human bocavirus infections. Clin Infect Dis 2008; 46: 547-9.

39.- Lindner J, Karalar L, Schimanski S, Pfister H, Struff W, Modrow S. Clinical and epidemiological aspects of human bocavirus infection. J Clin Virol 2008; 43: 391-5.
40.- Söderlund-Venermo M, Lahtinen A, Jartti T, Hedman L, Kemppainen K, Lehtinen P, et al. Clinical assessment and improved diagnosis of bocavirus-induced wheezing in children, Finland. Emerg Infect Dis 2009; 15: 1423-30.

41.- Don M, Söderlund-Venermo M, Valent F, Lahtinen A, Hedman L, Canciani M, et al. Serologically verified human bocavirus pneumonia in children. Pediatr Pulmonol 2010; 45: 120-6.

42.- Young N S, Brown K E. Parvovirus B19. N Engl J Med 2004; 350: 586-97. 